\title{
The emergence of Acinetobacter baumannii ST-6 multidrug resistant: A resilient biofilm producer, Malaysia
}

\author{
Jayakayatri Jeevajothi Nathan ${ }^{1}$, Mohd Nasir Mohd Desa ${ }^{2}$, Voon Chen Yong ${ }^{1}$, Parasakthi Navaratnam ${ }^{3}$, Navindra \\ Kumari Palanisamy ${ }^{4}$ and Eng Hwa Wong ${ }^{5 *}$ \\ ${ }^{1}$ School of Biosciences, Taylor's University, No.1, Jalan Taylor's, 47500 Subang Jaya, Selangor Darul Ehsan, Malaysia. \\ ${ }^{2}$ Department of Biomedical Sciences, Faculty of Medicine \& Health Sciences, Universiti Putra Malaysia, 43400 UPM \\ Serdang, Selangor Darul Ehsan, Malaysia. \\ ${ }^{3}$ School of Medicine and Health Sciences, Monash University Malaysia, Jalan Lagoon Selatan, Bandar Sunway, \\ 46150,Selangor Darul Ehsan, Malaysia. \\ ${ }^{4}$ Faculty of Medicine, Universiti Teknologi MARA (UiTM), $47000 \mathrm{Sg}$. Buloh, Selangor Darul Ehsan, Malaysia. \\ ${ }^{5}$ School of Medicine, Taylor's University, No.1, Jalan Taylor's, 47500 Subang Jaya, Selangor Darul Ehsan, Malaysia.
} Email: EngHwa.Wong@taylors.edu.my

Received 12 January 2016; Received in revised form 26 April 2016; Accepted 18 May 2016

\begin{abstract}
Aims: The aim of this research is to explore the presence of multidrug-resistance (MDR) Acinetobacter baumannii strains isolated from hospitalized patients in a tertiary-care center, Subang Jaya, Selangor, Malaysia and to compare their genotypic and phenotypic characteristics.

Methodology and results: Clonal relationships were determined by multilocus sequence typing (MLST) and biofilm forming ability was evaluated by using 2, 3 - bis (2 - methoxy - 4 - nitro - 5-sulfophenyl) - 5 - [(phenylamino) carbonyl] $2 \mathrm{H}$-tetrazolium hydroxide (XTT) reduction assay in microplates and Congo red agar method (CRA). Four virulence genes coding for $A$. baumannii pilus usher-chaperone assembly protein, csuE gene; outer membrane protein, ompA gene; biofilm poly- $\beta-1,6-$ Nacetylglucosamine (PNAG) synthesis protein, pgaA gene; and acinetobactin-mediated iron acquisition protein, bauA gene were searched for in a collection of strains. Antimicrobial resistance against 11 antibiotics were studied by broth microdilution method. Seventeen $A$. baumannii clinical strains were isolated and MLST showed that the strains belonged to 5 distinct sequence types (STs), namely, ST-6, ST-265, ST-324, ST-325 and ST-432. Fiftythree percent of the strains were resistant to 4 or more antibiotics. Twelve strains produced biofilm and out of them, 4 were strong biofilm producer, besides, these strong biofilm producers were MDR strains and belongs to ST-6. In addition, all strains were ompA positive, biofilm producing strains were csuE and pgaA positive and only strong biofilm producing strains were bauA positive.

Conclusion, significance and impact study: Our study demonstrates that the ST-6 strains in Malaysia could represent MDR, capable of forming strong biofilm and possess csuE, ompA, pgaA and bauA genes, virulence characteristics that probably help the bacteria to persist and cause infection.
\end{abstract}

Keywords: Acinetobacter baumannii, multilocus sequence typing, multidrug-resistant, biofilm

\section{INTRODUCTION}

Multidrug-resistant (MDR) $A$. baumannii is a rapidly emerging pathogen in healthcare settings and their increasing incidence is an alarming problem. Management of MDR $A$. baumannii infections is an area of great concern for clinicians. This bacterium is responsible for a wide spectrum of infections that include pneumonia, bacteremia, meningitis, urinary tract infection and wound infection (Maragakis and Perl, 2008). The episodes of infections caused by MDR $A$. baumannii becomes more challenging when the strains have the ability to form biofilm. Biofilm is described as "a complex aggregate of bacterial cells enclosed in a self-produced matrix of extracellular polymeric substance (EPS) adherent to an abiotic or biotic surface." It has been reported that $A$. baumannii can form biofilms on the surface which contribute to its ability to survive in a hospital setting and to persist for prolonged period (Manchanda et al., 2010). The aim of this study was to explore the presence of MDR in a collection of $A$. baumannii isolated from patients and to compare the following genotypic and phenotypic characteristics: multilocus sequence typing (MLST), virulence-associated factor-encoding genes (csuE, pgaA, ompA and bauA), biofilm formation and resistance profiles to 11 frequently 
used antibiotics. This will assist our clinicians in prescribing an appropriate antibiotic against infections caused by MDR biofilm producer strains.

\section{MATERIALS AND METHODS}

\section{Bacterial strains}

Seventeen clinical $A$. baumannii strains were collected from hospitalized patients in a tertiary-care hospital in Subang Jaya, Selangor, Malaysia between 2012 and 2013. Identification of the species were confirmed by API 20-NE system (BioMërieux, France). The strains were stored in Luria-Bertani (LB) broth with $15 \%$ glycerol at -80 ${ }^{\circ} \mathrm{C}$ until further characterization.

\section{MLST}

MLST based on the sequence of seven housekeeping genes (gltA, gyrB, gdhB, recA, cpn60, gpi and $r p o D$ ) were performed on all the strains as previously described (Qian et al., 2015). Briefly, all seven genes were amplified and sequenced and allele number and ST were assigned according to the scheme proposed in the MLST website (http://www.mlst.net/).

\section{Resistotyping}

All strains were tested for antibiotics resistance against a panel of 11 antimicrobials (polymyxin B, ampicillin/sulbactam, ceftazidime, meropenem, imipenem, amikacin, gentamicin, ciprofloxacin, levofloxacin, trimethoprim/sulfamethoxazole and tigecycline) using the broth microdilution following the guidelines recommended by Clinical and Laboratory Standards Institute guidelines (CLSI) M100-S24, 2014.

\section{Biofilm production assay}

Overnight cultures of $A$. baumannii grown in LB broth were washed twice with phosphate-buffered saline (PBS, $\mathrm{pH}$ 7.2) and diluted in PBS to obtain a suspension equivalent to 0.5 McFarland standards $\left(\sim 1 \times 10^{8}\right.$ $\mathrm{CFU} / \mathrm{mL}$ ). The suspension was then diluted to $1: 100$ in PBS. Acinetobacter baumannii ATCC $19606^{\top}$ was used as positive biofilm producing reference strains, whilst Pseudomonas aeruginosa ATCC 27853 was used as negative control. The experiment was performed in triplicate and repeated three times. Qualitative determination for the ability to form biofilm by the production of slime using Congo red agar (CRA) plate assay as described by Freeman et al., 1989. The CRA medium is composed of $30 \mathrm{~g} / \mathrm{L}$ Brain Heart Infusion (BHI) agar, $36 \mathrm{~g} / \mathrm{L}$ sucrose and $0.8 \mathrm{~g} / \mathrm{L}$ Congo red indicator. Sterilized aqueous Congo red stain was added to the media when the agar was cooled to $55^{\circ} \mathrm{C}$. Plates were then inoculated with test strains and were incubated aerobically for $24 \mathrm{~h}$ at $37^{\circ} \mathrm{C}$. Quantification of $A$. baumannii biofilms by XTT reduction assay was carried out by following the method described previously (Pierce et al., 2010). Briefly, $100 \mu \mathrm{L}$ cell suspensions were inoculated into wells of sterile 96-well flat-bottomed polystyrene microtitre plates (CTPP, USA). The plates were incubated for $24 \mathrm{~h}$ at $37^{\circ} \mathrm{C}$ under static conditions. After incubation, each well was carefully washed three times with $200 \mu \mathrm{L}$ PBS, dried and $100 \mu \mathrm{L}$ of saturated XTT solution was added, the plates were covered with aluminium foil and incubated at $37{ }^{\circ} \mathrm{C}$ for $2 \mathrm{~h}$. After incubation, $80 \mu \mathrm{L}$ of the XTT supernatant was transferred to a fresh 96-well plate and the calorimetric changes were measured at $490 \mathrm{~nm}$ using iMark $^{\mathrm{TM}}$ Microplate Absorbance Reader (Bio-Rad laboratories, Inc. USA). Results are reported according to published recommendations based on average optical density (OD) values and cut-off value (ODc) (ODc = average OD of negative control $+[3 \times$ standard deviation $(S D)$ of negative control]) (Abdi-Ali et al., 2014). Strains were divided into the following four categories: not a biofilm producer $(\mathrm{OD} \leq \mathrm{ODc})$; weak biofilm producer (ODc < OD $\leq 2 \times$ ODc); moderate biofilm producer $(2 \times \mathrm{ODc}<\mathrm{OD} \leq 4$ $\times \mathrm{ODc})$; and strong biofilm producer $(4 \times \mathrm{ODc}<\mathrm{OD})$.

Table 1: The sequences of the primers used with respective amplicon sizes in virulence characterization.

\begin{tabular}{llc}
\hline Genes & Primers & Amplicon size \\
\hline csuE & Forward: 5' CTTGGCTTTGGCGAACAT 3' & 207 \\
pgaA & Reverse: 5' ACTGCGGGCACAGAATAG 3' & 100 \\
& Forward: 5' AGGTTGATGTATTGCGTGAG 3' & 200 \\
ompA & Reverse: 5' TGCGGGTATTATTTAGC 3' & 303 \\
& Forward: 5' GACTACGGGCAAATCTCG 3' & 300 \\
bauA & Reverse: 5' ACTGGCTCACGGCACCTT 3' & \\
& Forward: 5' TAACACGAGGCATCAACC 3' & \\
\hline
\end{tabular}

\section{Virulotyping}

Virulence characterization on $A$. baumannii strains was performed by Polymerase Chain Reaction (PCR) in detection of csuE, pgaA, ompA and bauA genes associated in biofilm formation. Genomic DNA was extracted using DNA Extraction Kit (GeneAll Biotechnology Co., Ltd, Korea) following the manufacturer's guidelines. The DNA sequence of $c s u E$, pgaA, ompA and bauA s were obtained from the National Centre for Biotechnology Information (NCBI) GenBank (http://www.ncbi.nlm.nih.gov). Primer sequences were 
designed using Primer Premier 3 and were synthesized by Sigma Aldrich (Table 1). Annealing temperatures were optimized by gradient PCR and cycling conditions for each primer were as follows: initial denaturation $\left(95^{\circ} \mathrm{C}\right)$ for $3 \mathrm{~min}$; 35 cycles-denaturation $\left(95^{\circ} \mathrm{C}\right)$ for $30 \mathrm{sec}$, annealing $\left(60^{\circ} \mathrm{C}\right)$ for $30 \mathrm{sec}$ and extension $\left(72{ }^{\circ} \mathrm{C}\right)$ for 1 min; final extension $\left(72{ }^{\circ} \mathrm{C}\right)$ for $10 \mathrm{~min}$. All the amplified products were separated by electrophoresis in $1.3 \%$ agarose gels stained with GelRed ${ }^{\mathrm{TM}}$ Nucleic Acid Gel Stain (Biotium, Inc., USA). The agarose gel was viewed under UV light using imaging system (Alphalmager ${ }^{\circledR} \mathrm{HP}$, USA).

\section{RESULTS}

A total of 17 A. baumannii strains were tested in our study (Table 2). The strains were isolated from various body sites of infections from patients. Majority of the isolates were from wound swab $(n=10)$, followed by respiratory sites include sputum and tracheotomy site $(n=6)$ and blood $(n=1)$. MLST showed that the 17 strains belonged to five distinct STs: ST-6, ST-265, ST-324, ST-325 and ST-432. Fifty-three percent of the $A$. baumannii strains were resistant to at least four of the eleven tested antibiotics: imipenem, meropenem, gentamicin and amikacin. Likewise, four strains were resistant to nine out of the eleven antibiotics tested: ampicillin/sulbactam, ceftazidime, imipenem, meropenem, gentamicin, amikacin, ciprofloxacin, levofloxacin and trimethoprim/ sulfamethoxazole. These four strains were classified as MDR [strains resistant to at least three classes of antimicrobial agents - all penicillins and cephalosporins (including inhibitor combinations), fluoroquinolones, and aminoglycosides] (Manchanda et al., 2010). All the MDR strains were belonged to ST-6. A good correlation was observed between the MLST results and antibiotic resistant pattern. Of them, 4 strains showed black colonies (strong biofilm producer), 8 strains showed black colonies with red center (moderate/weak biofilm producer) and 5 strains showed red colonies (non-biofilm producers) (Figure 1). These CRA results were validated with XTT reduction assay. XTT reduction assay showed that 12 strains were biofilm producer (4 strains - strong biofilm producer; 4 strains - moderate biofilm producer; 4 strains weak biofilm producer). All $A$. baumannii strains tested positive for the ompA gene, which codes for outer membrane protein of $A$. baumannii. Conversely, all biofilm producing strains harbored the csuE and pgaA genes, coding for pilus usher-chaperone assembly protein and biofilm PNAG synthesis protein respectively. Interestingly, only strong biofilm producer strains tested positive for bauA gene, coding for acinetobactin-mediated iron acquisition protein. In addition, these strong biofilm producer were of the MDR strains.
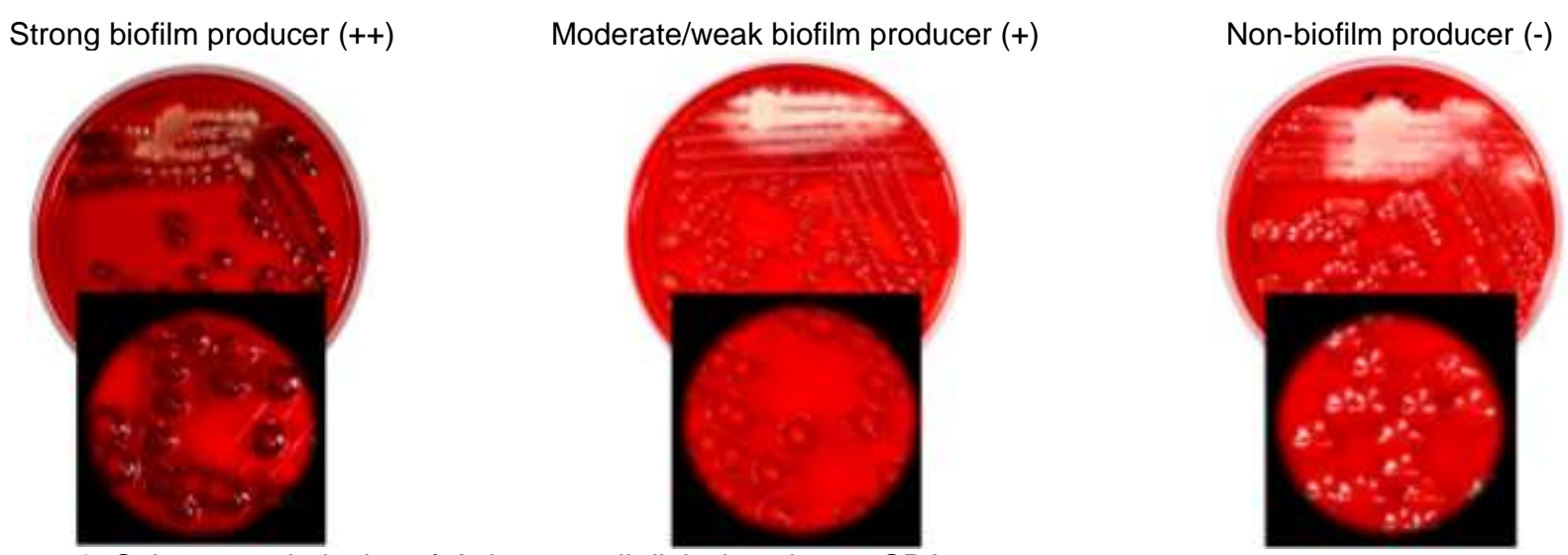

Figure 1: Colony morphologies of $A$. baumannii clinical strains on CRA.

\section{DISCUSSION}

Acinetobacter baumannii has emerged as leading opportunistic pathogens and nosocomial pathogen worldwide (Allen and Hartman, 2009). MDR A. baumannii emergence could therefore complicate the treatment, leading to recurrent disease. Four of the seventeen tested strains (23.5\%) were MDR. According to MLST results, these four MDR strains were belong to ST-6. To our knowledge, this is the first report of the ST-6 in Malaysia, ST-6 was originally reported to belong to Barcelona epidemic strains that was responsible for the outbreak in Elche General Hospital during 2001 and 2002 (Bartual et al., 2005). In addition, Bartual et al. (2005) has also reported that the ST-6 to be a clonal lineage of the
European clone II, which highlights the widespread of this successful epidemic clone during the past two decades. The MDR A. baumannii appears to play an significant role in the remarkable ability to persist and spread in the hospital settings, together with its ability to colonize both abiotic and biotic surfaces and to grow as biofilm has been studied (Eijkelkamp et al., 2011; Gurung et al., 2013). In agreement with previously published results on MDR A. baumannii, we found that MDR A. baumannii are good biofilm producers (Eijkelkamp et al., 2011; Gurung et al., 2013). This feature could confer advantages in persistence and spreading of MDR $A$. baumannii ST-6 in the hospital settings. Concerning virulotyping, all strains possessed the ompA gene, however only biofilm producing strains possessed $c s u E, p g a A$ and surprisingly 
Malays. J. Microbiol. Vol 12(4) 2016, pp. 265-269

Table 2: Acinetobacter baumannii strains used in the present study and their characteristics.

\begin{tabular}{|c|c|c|c|c|c|c|c|c|c|c|c|c|c|c|c|c|c|c|c|c|}
\hline \multirow[t]{2}{*}{ Strain } & \multirow[t]{2}{*}{$\begin{array}{l}\text { Year of } \\
\text { isolation }\end{array}$} & \multirow[t]{2}{*}{ Source } & \multirow[t]{2}{*}{ ST } & \multicolumn{11}{|c|}{ Resistotype } & \multicolumn{2}{|c|}{$\begin{array}{l}\text { Biofilm } \\
\text { Phenotype }\end{array}$} & \multicolumn{4}{|c|}{ Virulotype $^{d}$} \\
\hline & & & & $\sum_{<}^{\infty}$ & $\frac{N}{\delta}$ & $\Sigma$ & $\frac{n}{\stackrel{n}{\Sigma}}$ & $\stackrel{m}{a}$ & Z & $\stackrel{y}{<}$ & O্ত & $\frac{0}{0}$ & 岂 & 文 & $\mathrm{CRA}^{\mathrm{b}}$ & $\begin{array}{l}\text { XTT } \\
\text { reduction } \\
\text { assayc }^{c}\end{array}$ & csuE & pgaA & ompA & bauA \\
\hline$A B-1$ & 2012 & Sputum & 265 & $S$ & $S$ & $\overline{\mathrm{R}}$ & $\mathrm{R}$ & $S$ & $\mathrm{R}$ & $\mathrm{R}$ & $S$ & $S$ & $\vec{S}$ & $S$ & - & - & - & - & + & - \\
\hline AB-2 & 2012 & Sputum & 265 & $S$ & $S$ & $\mathrm{R}$ & $\mathrm{R}$ & S & $\mathrm{R}$ & $\mathrm{R}$ & $S$ & $S$ & $S$ & S & - & - & - & - & + & - \\
\hline AB-3 & 2012 & Sputum & 265 & $S$ & $S$ & $\mathrm{R}$ & $\mathrm{R}$ & $S$ & $\mathrm{R}$ & $\mathrm{R}$ & $S$ & $S$ & $S$ & S & - & - & - & - & + & - \\
\hline AB-4 & 2012 & Wound & 325 & $S$ & $S$ & $S$ & $S$ & $S$ & $S$ & $S$ & $S$ & $S$ & $S$ & $S$ & + & ++ & + & + & + & - \\
\hline$A B-5$ & 2012 & Sputum & 265 & $S$ & $S$ & $\mathrm{R}$ & $\mathrm{R}$ & $S$ & $\mathrm{R}$ & $\mathrm{R}$ & $S$ & $S$ & $S$ & S & - & - & - & - & + & - \\
\hline AB-6 & 2012 & $\begin{array}{l}\text { Wound } \\
\text { swab }\end{array}$ & 325 & S & S & S & $S$ & $S$ & S & $S$ & S & $\mathrm{S}$ & $S$ & S & + & ++ & + & + & + & - \\
\hline AB-7 & 2012 & $\begin{array}{l}\text { Wound } \\
\text { swab }\end{array}$ & 6 & $\mathrm{R}$ & $\mathrm{R}$ & $\mathrm{R}$ & $\mathrm{R}$ & $S$ & $\mathrm{R}$ & $\mathrm{R}$ & $S$ & $\mathrm{R}$ & $\mathrm{R}$ & $\mathrm{R}$ & ++ & +++ & + & + & + & + \\
\hline AB-8 & 2012 & $\begin{array}{l}\text { Wound } \\
\text { swab }\end{array}$ & 6 & $\mathrm{R}$ & $\mathrm{R}$ & $\mathrm{R}$ & $\mathrm{R}$ & $S$ & $\mathrm{R}$ & $\mathrm{R}$ & $\mathrm{S}$ & $\mathrm{R}$ & $\mathrm{R}$ & $\mathrm{R}$ & ++ & +++ & + & + & + & + \\
\hline AB-9 & 2012 & $\begin{array}{l}\text { Wound } \\
\text { swab }\end{array}$ & 325 & $S$ & $\mathrm{~S}$ & $S$ & $S$ & $S$ & $S$ & $S$ & $\mathrm{~S}$ & $\mathrm{~S}$ & $S$ & S & + & ++ & + & + & + & - \\
\hline$A B-10$ & 2012 & $\begin{array}{l}\text { Wound } \\
\text { swab }\end{array}$ & 432 & $S$ & $\mathrm{~S}$ & $S$ & $S$ & $S$ & $S$ & $S$ & $\mathrm{~S}$ & $\mathrm{~S}$ & $S$ & S & + & + & + & + & + & - \\
\hline$A B-11$ & 2012 & Blood & 324 & $S$ & $\mathrm{~S}$ & $S$ & $S$ & $S$ & $S$ & $S$ & $S$ & $S$ & $S$ & $S$ & + & + & + & + & + & - \\
\hline AB-12 & 2012 & $\begin{array}{l}\text { Wound } \\
\text { swab }\end{array}$ & 432 & $S$ & $S$ & $S$ & $S$ & $S$ & $S$ & $S$ & $S$ & $S$ & $S$ & $S$ & + & + & + & + & + & - \\
\hline$A B-13$ & 2012 & $\begin{array}{l}\text { Tracheotomy } \\
\text { site }\end{array}$ & 6 & $\mathrm{R}$ & $\mathrm{R}$ & $\mathrm{R}$ & $\mathrm{R}$ & $S$ & $\mathrm{R}$ & $\mathrm{R}$ & $S$ & $\mathrm{R}$ & $\mathrm{R}$ & $\mathrm{R}$ & ++ & +++ & + & + & + & + \\
\hline$A B-14$ & 2013 & $\begin{array}{l}\text { Wound } \\
\text { swab }\end{array}$ & 6 & $\mathrm{R}$ & $\mathrm{R}$ & $\mathrm{R}$ & $\mathrm{R}$ & $S$ & $\mathrm{R}$ & $\mathrm{R}$ & S & $\mathrm{R}$ & $\mathrm{R}$ & $\mathrm{R}$ & ++ & +++ & + & + & + & + \\
\hline$A B-15$ & 2013 & $\begin{array}{l}\text { Wound } \\
\text { swab }\end{array}$ & 432 & $S$ & $S$ & $S$ & $S$ & $S$ & $S$ & $S$ & S & $\mathrm{S}$ & $S$ & $S$ & + & + & + & + & + & - \\
\hline AB-16 & 2013 & Sputum & 265 & $S$ & S & $\mathrm{R}$ & $\mathrm{R}$ & $S$ & $\mathrm{R}$ & $\mathrm{R}$ & $S$ & $\mathrm{~S}$ & $S$ & $S$ & - & - & - & - & + & - \\
\hline AB-17 & 2013 & $\begin{array}{l}\text { Wound } \\
\text { swab }\end{array}$ & 325 & $S$ & $S$ & $S$ & $S$ & $S$ & $S$ & $S$ & $S$ & $S$ & $S$ & $S$ & + & ++ & + & + & + & - \\
\hline
\end{tabular}

${ }^{2}$ Resistotype against a panel of 11 known antibiotics were determined using the broth microdilution method. AMS, ampicillin/sulbactam; CAZ, ceftazidime; IMI, imipenem; MRP, meropenem; PB, polymyxin B; CN, gentamicin; AK, amikacin; TGC, tigecycline; CIP, ciprofloxacin; LEV, levofloxacin; SXT, trimethoprim/sulfamethoxazole; S, susceptible; R, resistant.

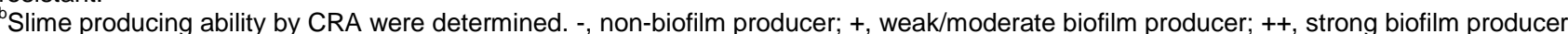

${ }^{\circ}$ Biofilm forming ability by XTT reduction assay were determined. -, non-biofilm producer; +, weak biofilm producer; ++, moderate biofilm producer; +++, strong biofilm producer.

${ }^{\mathrm{d} P C R}$ results using primers csuE, pgaA, ompA and bauA genes. -, absent; +, presence. 
only strong biofilm producers possessed bauA genes. This bauA gene coding for acinetobactin-mediated iron acquisition protein may also help the bacteria to be more virulent and to persist. CRA method to detect biofilm production was performed in this study. It is known that Congo red can directly interact with certain polysaccharide forming colored complexes. In the present study, $12(70.6 \%)$ of the total $A$. baumannii isolates showed biofilm production by CRA method which was verified and supported by the most reproducible, accurate and efficient XTT reduction assay (Taff et al., 2012). The use of rapid, simple, inexpensive method like CRA method is thus an urgent priority for determining the biofilm producing ability which is feasible for low resources countries. Microbial biofilms lead to chronic infections and are a major challenge for the clinicians and have economic significance. Hence, detection of biofilm producers, judicious antibiotic usage and strict appliance of hospital infection control practices can assist in the prevention of such hitches.

In conclusion, this study provides an insight into MDR A. baumannii by demonstrating that: (i) ST-6 could represent MDR A. baumannii in Malaysia; (ii) All MDR $A$. baumannii in our study are strong biofilm producers and possess the bauA gene coding for acinetobactin-mediated iron acquisition protein.

\section{CONFLICT OF INTEREST}

None to declare.

\section{ACKNOWLEDGEMENTS}

This work was supported by Taylor's University through its PhD Scholarship Programme. This research was also financially supported by the Fundamental Research Grant Scheme (FRGS/1/2011/SKK/TAYLOR/02/1) from the Ministry of Higher Education (MOHE, Putrajaya, Malaysia). The authors acknowledge Taylor's University for providing research facilities.

\section{REFERENCES}

Abdi-Ali, A., Hendiani, S., Mohammadi, P. and Gharavi, S. (2014). Assessment of biofilm formation and resistance to imipenem and ciprofloxacin among clinical isolates of Acinetobacter baumannii in Tehran. Jundisphapur Journal of Microbiology 7, e8606.

Allen, D. M. and Hartman, B. J. (2009). Acinetobacter species. In: Principles and practice of infectious diseases. Mandell, G. L., Bennet, J. E. and Doun, R. (eds.). Elsevier Churchill Livingstone, Philadelphia. pp. 2881-2885.

Bartual, S. G., Seifert, H., Hippler, C., Luzon, M. A., Wisplinghoff, H. and Rodriguez-Valera, F. (2005). Development of a multilocus sequence typing scheme for characterization of clinical isolates of Acinetobacter baumannii. Journal of Clinical Microbiology 43, 43824390.
Clinical Laboratory Standards Institute. (2014). Performance standards for antimicrobial susceptibility testing; $24^{\text {th }}$ informational supplement, CLSI M100S24.

Eijkelkamp, B. A., Stroeher, U. H., Hassan, K. A., Papadimitrious, M. S., Paulsen, I. T. and Brown, M. H. (2011). Adherence and motility characteristics of clinical Acinetobacter baumannii isolates. FEMS Microbiology Letters 323, 44-51.

Freeman, D. J., Falkner, F. R. and Keane, C. T. (1989). New method for detecting slime production by coagulase-negative staphylococci. Journal of Clinical Pathology 42, 872-874.

Gurung, J., Khyriem, A. B., Banik, A., Lyngdoh, W. V., Choudhury, B. and Bhattacharyya, P. (2013). Association of biofilm production with multidrug resistance among clinical isolates of Acinetobacter baumannii and Pseudomonas aeruginosa from intensive care unit. Indian Journal of Critical Care Medicine 17, 214-218.

Manchanda, V., Sanchaita, S. and Singh, N. P. (2010). Multidrug resistant Acinetobacter. Journal of Global Infectious Diseases 2, 291-304.

Maragakis, L. L. and Perl, T. M. (2008). Acinetobacter baumannii: Epidemiology, antimicrobial resistance, and treatment options. Clinical Infectious Diseases 46, 1254-1263.

Pierce, C. G., Uppuluri, P., Tummala, S. and LopezRibot, J. L. (2010). A 96 well microtiter plate based method for monitoring formation and antifungal susceptibility testing of Candida albicans biofilms. Journal of Visualized Experiments 44, pii:2287.

Qian, Y., Dong, X., Wang, Z., Yang, G. and Liu, Q. (2015). Distributions and types of multidrug-resistant Acinetobacter baumannii in different departments of a general hospital. Jundishapur Journal of Microbiology 8, e22935.

Taff, H. T., Nett, J. E. and Andes, D. R. (2012). Comparative analysis of Candida biofilm quantification assays. Medical Mycology 50, 214-218. 